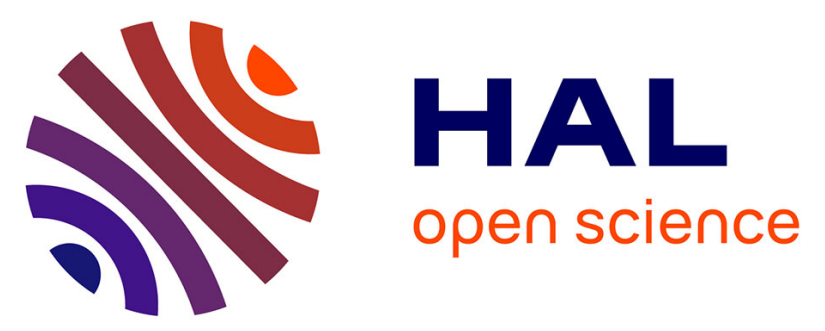

\title{
Kinetics and mechanism of polymorphic transformation of sorbitol under mechanical milling
}

\author{
Anthony Dupont, Mathieu Guerain, Florence Danede, Laurent Paccou, \\ Yannick Guinet, Alain Hédoux, Jean-François Willart
}

\section{To cite this version:}

Anthony Dupont, Mathieu Guerain, Florence Danede, Laurent Paccou, Yannick Guinet, et al.. Kinetics and mechanism of polymorphic transformation of sorbitol under mechanical milling. International Journal of Pharmaceutics, 2020, International Journal of Pharmaceutics, 590, pp.119902. 10.1016/j.ijpharm.2020.119902 . hal-02965510

\section{HAL Id: hal-02965510 \\ https://hal.univ-lille.fr/hal-02965510}

Submitted on 13 Oct 2020

HAL is a multi-disciplinary open access archive for the deposit and dissemination of scientific research documents, whether they are published or not. The documents may come from teaching and research institutions in France or abroad, or from public or private research centers.
L'archive ouverte pluridisciplinaire HAL, est destinée au dépôt et à la diffusion de documents scientifiques de niveau recherche, publiés ou non, émanant des établissements d'enseignement et de recherche français ou étrangers, des laboratoires publics ou privés. 


\title{
Kinetics and mechanism of polymorphic transformation of sorbitol under mechanical milling
}

\author{
Anthony Dupont, Mathieu Guerain, Florence Danède, Laurent Paccou, Yannick Guinet \\ Alain Hédoux and Jean-François Willart*
}

Univ. Lille, CNRS, INRAE, Centrale Lille, UMR 8207

UMET - Unité Matériaux et Transformations, F-59000 Lille, France

*Corresponding author: jean-francois.willart@univ-lille.fr

\begin{abstract}
:
In this paper, we present a kinetic investigation of the polymorphic transformation $\gamma \rightarrow \alpha$ of sorbitol under milling in the objective to identify the microscopic mechanisms that govern this type of solidstate transformation. The milling was performed with a high energy planetary mill and the milled material was analysed by DSC, PXRD and Raman spectrometry. The transformation kinetics was found to be sigmoidal with a noticeable incubation time. Moreover, this incubation time was shown to shorten rapidly when seeding the initial form $\gamma$ with the final form $\alpha$. The origin of the incubation period and its evolution upon seeding are puzzling as polymorphic transformations induced by milling are not expected to occur through a nucleation and growth process. To explain these puzzling kinetic features, we propose a two-step transformation mechanism involving local amorphisations due to the mechanical impacts, immediately followed by rapid recrystallizations of the amorphized fractions. The key point of the mechanism is that recrystallizations are oriented towards the forms $\gamma$ or $\alpha$, depending on the crystalline form of neighbouring crystallites. This mechanism has been validated by numerical simulations which were able to reproduce all the experimental kinetic features of the polymorphic transformation (kinetic law and effects of seeding) upon milling.
\end{abstract}




\section{INTRODUCTION}

It is now well recognized that mechanical milling can induce amorphizations or polymorphic transformations of pharmaceuticals ${ }^{1,2}$. Interestingly, these transformations occur in the solid state so that they can be used to drive drugs into specific physical states which could hardly be obtained by other processing techniques using melting and dissolution stages. Milling can be used, for instance, to amorphize drugs without chemical changes ${ }^{3,4}$ (mutarotation, thermal degradation...), to produce glass solutions without using solvents ${ }^{5-7}$, to produce glass solutions of immiscible compounds ${ }^{8}$, to explore the polymorphism of drugs ${ }^{9-11}$, to form co-crystals ${ }^{12-15} \ldots$. However, we also have to be aware that milling is widely used in the manufacturing processes to handle the microstructure of drug powders ${ }^{16-}$ ${ }^{20}$ and in particular to reduce the particle size. In this case, it can thus induce undesired physical state transformations which can modify the therapeutic properties of drugs. As a result, whether we want to induce or avoid them, transformations under milling must be fully understood to be perfectly controlled.

In the last decades many investigations have been dedicated to the rationalization of milling induced transformations ${ }^{2,21}$. They have shown the key role of some milling parameters like the milling temperature ${ }^{22,23}$, the milling intensity ${ }^{24}$ and the milling atmosphere ${ }^{25}$ on the nature of the transformations induced by milling. From a general point of view, it appears that the nature of the transformation is mainly driven by the relative position of the milling temperature with respect to the glass transition temperature $\left(T_{\mathrm{g}}\right)$ of the materia| ${ }^{26,27}$. Amorphizations mainly occur when the milling is performed below the glass transition temperature $\left(T_{\mathrm{g}}\right)$ of the material ${ }^{23,28-31}$ while polymorphic transformations generally occur when the milling is performed above $\mathrm{T}_{\mathrm{g}}{ }^{32-34}$.

While macroscopic parameters driving amorphizations and polymorphic transformations upon milling are quite clearly identified, little is known about the microscopic mechanisms by which these two types of transformations operate. The difficulty mainly arises from the heterogeneous character of the powder during the transformation ${ }^{35}$. For instance, in the case of ball milling, the transformation of a grain generally requires several impacts which occur randomly during the milling process. Moreover, the physical state generated upon milling is generally not the most stable so that physical transformations toward a more stable state can possibly occur during the laps time between two mechanical shocks. For instance, it is not yet clear if polymorphic transformations induced by milling occur directly or if they involve, on the contrary, a transient amorphization stage ${ }^{26}$ immediately followed by a rapid recrystallization. Interestingly, all this complexity must have a direct repercussion on the kinetics of milling induced transformations ${ }^{36}$. As a result, detailed analysis of these kinetics can potentially enlighten the microscopic mechanisms of these transformations. Up to now, only few kinetics of transformation have been reported in the literature but their inspection already suggests interesting comments. As sketched in figure 1, it appears that amorphization kinetics, generally obey an exponential relaxation law (e.g. trehalose ${ }^{37}$, chlorhexidine ${ }^{9}$, dexamethasone ${ }^{10}$, lactulose ${ }^{38} \ldots$ ) while kinetics of polymorphic transformations have generally a sigmoidal shape characterized by a long incubation time preceding a fast transformation stage (e.g. mannitol ${ }^{32}$, chloramphenicol palmitate ${ }^{39} \ldots$..). The origin of this striking difference remains an open question. Moreover, the physical mechanisms which are responsible for the quite long incubation preceding the polymorphic transformations are not yet understood. Investigations of these striking kinetic features are thus expected to provide interesting information concerning the microscopic mechanisms which govern these transformations. 
In this paper, we present experimental and numerical investigations of the kinetics of the milling induced polymorphic transformation $\gamma \rightarrow \alpha$ of sorbitol which was detected in previous papers ${ }^{40,41}$. The nature of this transformation is coherent with the fact that sorbitol has a glass transition $\left(T_{g}=-3^{\circ} \mathrm{C}^{42,43}\right)$ located noticeably below the milling temperature (RT). The forms $\gamma$ and $\alpha$ have the advantage to be characterized by well separated meting points and noticeably different crystalline structures so that the structural evolution of sorbitol upon milling can be followed easily and accurately by both calorimetric (DSC) and structural (XRD and RAMAN) techniques. The main objective is here to precise the microscopic mechanisms which govern the transformation $\gamma \rightarrow \alpha$ upon milling. In particular, two open questions are addressed: (i) Does this polymorphic transformation involves a transient amorphization stage? (ii) What is the origin of the induction period which precedes the effective transformation and gives the kinetics a sigmoidal shape? The experimental investigations were performed by DSC, XRD and Raman spectroscopy. The numerical investigations were performed by simulation of a phenomenological model ${ }^{32}$ which captures the essential features of both the experimental system and the milling perturbation.

\section{EXPERIMENTALS}

$\gamma$-sorbitol was purchased from SIGMA Aldrich (purity higher than 99.5\%) and used without further purification.

Ball milling was performed in a high energy planetary mill (Pulverisette, 7-Fritsch) at room temperature. We used $\mathrm{ZrO}_{2}$ milling jars of $43 \mathrm{~cm}^{3}$ with seven balls (diameter $15 \mathrm{~mm}$ ) of the same material. The rotation speed of the solar disk was set to $400 \mathrm{rpm}$ which corresponds to an average acceleration of the milling balls of $5 \mathrm{~g}\left(\mathrm{~g}=9.81 \mathrm{~m} \cdot \mathrm{s}^{-2}\right.$ is the acceleration of gravity). We took care to alternate milling periods ( $10 \mathrm{~min}$ ) with pause periods $(5 \mathrm{~min})$ in order to limit the mechanical heating of the sample.

Powder X-ray diffraction (PXRD) experiments were performed with a Panalytical XPert PRO diffractometer (Almlo, The Netherlands), equipped with a copper X-ray tube $(\lambda=0.15406 \mathrm{~nm})$ and a linear detector allowing acquisition of diffraction pattern from $2 \theta=4^{\circ}$ to $2 \theta=35^{\circ}$ with a scan step of $0.0167 \%$. Powders are placed in a rotating aluminium sample holder $(13 \mathrm{~mm}$ diameter and $2 \mathrm{~mm}$ depth) in rotation ( $15 \mathrm{rpm}$ ) to improve statistics.

Structural analyses were carried out using the Rietveld method ${ }^{44}$ on each diffraction diagram. It consists in calculating a theoretical diffraction pattern that best fits experimental data. Starting from a reference structure ${ }^{45,46}$, a refinement of the various parameters of the simulated function is performed in order to minimize the weighted profile R-factor $\left(R_{w p}\right)$ defined by Equation (1):

$$
R w p=\left[\frac{\sum_{i}\left[w_{i}\left(I_{i}^{\text {exp }}-I_{i}^{\text {sim }}\right]^{2}\right.}{\sum_{i}\left(w_{i} I_{i}^{\text {exp }}\right)^{2}}\right]^{1 / 2} \text { with } w_{i}=\frac{1}{\sqrt{I_{i}^{\text {exp }}}}
$$


where $I_{i}^{\text {exp }}$ is the intensity of the experimental diffraction pattern at point $\mathrm{i}$ along the diffraction pattern and $I_{i}^{s i m}$ is the intensity of the simulated diffraction pattern at point I, depending on the crystal structure (lattice parameters, atomic positions...). For details on the simulation of the diffraction pattern, see ref ${ }^{44}$. It is generally considered that a $R_{w p}$ ratio below $10 \%$ is acceptable ${ }^{44}$. The position and the shape of Bragg peaks depend on lattice parameters and structural defects. In particular, the width of the diffraction peaks is the parameter that contains information about the average crystallite size and the microstrain of constitutive crystallites. These structural parameters can be included in the simulation profile and optimized to match the experimental diagram. For each diagram, two refinements were performed from slightly different starting parameters to evaluate the robustness of the resulting solution. The reference structures and the starting parameters $I_{i}^{\text {sim }}$ used for the calculation are those determined by Rukiah et al. ${ }^{45}$ for the $\gamma$ form and Park et al. ${ }^{46}$ for the $\alpha$ form. Both structures are orthorhombic and their lattice parameters are reported in the Table 1 . Rietveld analyses were carried out with the $\mathrm{MAUD}^{47}$ software on the whole diagram. The Bragg peak profile corresponding to the instrumental resolution, was independently determined with the Rietveld analysis using NAC and silicon references. It was then implemented in MAUD and used to determine the structural composition of sorbitol ( $\alpha$ and $\gamma$ ) during the milling process.

Differential scanning calorimetry (DSC) experiments were performed with a Q200 microcalorimeter of TA Instruments (Guyancourt, France). During all the measurements, the calorimeter head was flushed with highly pure nitrogen gas. Temperature and enthalpy readings were calibrated using pure indium at the same scan rates used in the experiments. All heating DSC scans reported in the figures were performed at the rate of $2^{\circ} \mathrm{C} / \mathrm{min}$. The samples have been placed in open pans (pans with no lid) to allow any water absorbed during the milling process to evaporate upon heating. Small sample size (typically $4 \mathrm{mg}$ ) was used to improve the resolution and the thermal conductivity. Thermograms were analysed using the "Universal Analysis" Software from TA Instruments.

Raman spectroscopy experiments were performed using a InVia Renishaw micro-Raman spectrometer. The laser line (785 nm line from a Renishaw laser diode) was focused on the powder sample via a Leica $X 50$ objective providing the signal within a volume of about $250{\mu \mathrm{m}^{3}}^{3}$. The acquisition time for collecting Raman spectra in the molecular fingerprint region $\left(800-1500 \mathrm{~cm}^{-1}\right)$ was typically $60 \mathrm{~s}$. Spectra were baseline corrected and normalized by the integrated intensity of the most intense and isolated band of the spectral region around $880 \mathrm{~cm}^{-1}$.

Numerical simulations were performed with a PC and the code was written in visual basic using the Visual Studio software. 


\section{RESULTS AND ANALYSIS}

\subsection{KINETICS OF POLYMORPHIC TRANSFORMATION}

Figure 2a shows $X$-ray diffraction patterns of sorbitol recorded after different milling times $\left(t_{m}\right)$ ranging from 0 to $300 \mathrm{~min}$. At $t_{m}=0$ min the diffractogram only shows the Bragg peaks characteristic of the form $\gamma$ of sorbitol. From $t_{m}=0$ to $90 \mathrm{~min}$ there is no change in the $\mathrm{X}$-ray diffraction pattern. There are in particular no new Bragg peaks nor any extra background. This indicates that neither polymorphic transformation nor significant amorphization induced by the milling process can be detected during this milling period. However, one can note a slight broadening of the Bragg peaks signalling a slight reduction of the size of the crystallites which constitute the powder grains. From $t_{m}=90$ to $180 \mathrm{~min}$ we observe the progressive disappearance of Bragg peaks associated with the $\gamma$ form and the development of new Bragg peaks characteristic of the metastable polymorphic form $\alpha$. This indicates that a polymorphic transformation $\gamma \rightarrow \alpha$ has occurred during this milling period. At $t_{m}=180$ min, no more trace of the phase $\gamma$ can be observed indicating that the conversion is complete. No more transformation could be detected for longer milling times (e.g. for $t_{m}=300 \mathrm{~min}$ ). Moreover, the form $\alpha$ obtained by milling is unstable and thus expected to transforms back toward the stable form $\gamma$ after the end of the milling. However, this reverse transformation was found to be slow and no sign of form $\gamma$ could be detected during the first 24 hours after the end of the milling. As a result, the reverse transformation $\alpha \rightarrow \gamma$ during the milling pauses ( $5 \mathrm{~min}$ ) and the time lag between the end of the milling and the analysis of the sample (20-30 $\mathrm{min})$ is expected to have a negligible effect on the kinetics of the transformation $\gamma \rightarrow \alpha$ induced by milling.

The heating DSC scans $\left(+2^{\circ} \mathrm{C} / \mathrm{min}\right)$ of milled compounds are reported in Figure $2 \mathrm{~b}$. The un-milled sample $\left(t_{m}=0 \mathrm{~min}\right.$ ) shows an endotherm at $\mathrm{T}_{\mathrm{m}}=98.5^{\circ} \mathrm{C}$ corresponding to the melting of the form $\gamma$. The enthalpy of melting $\left(\Delta \mathrm{H}_{\mathrm{m}}=175 \mathrm{~J} / \mathrm{g}\right)$ is close to those previously reported in the literature ${ }^{48}$. From $t_{m}=0$ to $90 \mathrm{~min}$, we observe a slight and progressive shift of the melting peak toward the low temperatures and a slight decrease of the melting enthalpy. After 90 min of milling, the melting point is depressed by more than $5^{\circ} \mathrm{C}$ and $\Delta \mathrm{H}_{\mathrm{m}}$ is lowered by about $20 \%(142 \mathrm{~J} / \mathrm{g})$ in regard to the non-milled sample. This behaviour is due to both a strong crystallite size reduction ${ }^{49}$ and crystal defects generated by the milling process ${ }^{50}$. After 140 min of milling, the melting peak of the form $\gamma$ drops while a second endothermic peak is clearly detected around $85^{\circ} \mathrm{C}$. It corresponds to the melting of the form $\alpha$, previously detected in the X-ray diffraction experiments of figure $2 \mathrm{a}$. It must be noted that the two endotherms are separated by a small exotherm attributed to the recrystallization of the freshly melted form $\alpha$ toward the form $\gamma$. The overlapping of the three enthalpic events prevents an accurate determination of the fractions of forms $\gamma$ and $\alpha$ from their melting enthalpies. For longer milling $\left(t_{m}>\right.$ $140 \mathrm{~min}$ ), the melting peak of form $\alpha$ further develops while that corresponding to form $\gamma$ progressively vanishes. This confirms the polymorphic transformation $\gamma \rightarrow \alpha$ previously detected by X-ray diffraction. After 180 min of milling the melting peak of form $\gamma$ has totally disappeared indicating the completion of the transformation. The temperature and enthalpy of melting of form $\alpha\left(T_{m}=86.2{ }^{\circ} \mathrm{C}, \Delta \mathrm{H}_{\mathrm{m}}=168\right.$ $\mathrm{J} / \mathrm{g}$ ) produced by milling are slightly lower than those previously reported in the literature for a nonmilled sample ${ }^{48}$. This is due to the small crystallite size and crystal defects arising from the milling ${ }^{49,50}$. We can also note the disappearance of the intermediate recrystallization of the melt toward the form $\gamma$ for milling times longer than $140 \mathrm{~min}$. In fact, this recrystallization is expected to be facilitated by the presence of remaining $\gamma$-crystallites which act as seeds for crystallization. Since for increasing milling 
times the number of $\gamma$-crystallites decreases, the recrystallization propensity of the melt toward this phase upon heating also decreases and then vanishes.

The XRD patterns recorded during the 300 min milling process have been analysed thanks to the Rietveld method. The $R_{w p}$ factors are systematically below $10 \%$, indicating the very good correlation between calculated and experimental diagrams, as it can be seen in the example of figure 3 . This analyse gives directly access to the fractions of forms $\alpha$ and $\gamma$ after each milling time and thus, to the kinetics of the transformation $\gamma \rightarrow \alpha$ as reported in figure 4. This kinetics is characterized by an incubation time of nearly 120 min followed by a rapid transformation toward the form $\alpha$ completed in less than one hour. This gives the kinetics a sigmoidal shape characteristic of polymorphic transformations induced by milling ${ }^{32}$.

The long incubation time which precedes the effective transformation is puzzling and its origin remains an open question. A few papers ${ }^{26,51-53}$ have suggested that polymorphic transformations induced by ball milling involve a transient amorphization of impacted fractions, immediately followed by a fast recrystallization. Recently, it was proposed ${ }^{32}$ that the nature of this recrystallization (toward the initial form or toward to final form) is driven by a seeding effect induced by the surrounding crystallites. The competition between the two kinds of recrystallization was shown to be responsible for a stationary state where a large fraction of crystallites of the starting form coexists with a small fraction of short living crystallites of the final form. This mechanism is expected to be responsible for the initial incubation time which characterizes kinetics of polymorphic transformations. The small fraction of short living crystallites of the final form present during the incubation time could be revealed and quantified in the case of the polymorphic transformation of mannitol ${ }^{32}$. This was done using synchrotron X-ray diffraction in the course of an in situ milling experiment (i.e. during the milling itself). The transformation then really starts when some crystallites of the final form accidentally group together to make a cluster. Such a clustering has a stabilizing effect since, in case of amorphization of one of them by a mechanical shock, its recrystallization is likely to occur toward the same crystalline form than that of neighbouring crystallites. This cluster will then favour the transformation of neighbouring crystallites of the starting form to rapidly induce a total conversion of the sample.

In the present case of sorbitol, no sign of the final form $\alpha$ could be detected during the incubation stage. However, it is possible that the classical X-ray diffraction used for analysing the milled sample is not sensitive enough to detect such a small fraction of form $\alpha$. It is also possible that some reverse transformation of the form $\alpha$ toward the more stable form $\gamma$ occurs during the laps time between the sampling of the sample in the milling jar and its analysis by PXRD making the form $\alpha$ even more difficult to detect. To overcome these detection limits and reveal the presence of a tiny fraction of $\alpha$-crystallites during the incubation time, we have studied the kinetics of polymorphic transformation of sorbitol in physical mixtures of $\gamma$ and $\alpha$ forms. Figure 5 shows the kinetics of transformation $\gamma \rightarrow \alpha$ determined by PXRD during the milling of physical mixtures of $\gamma$-sorbitol and $\alpha$-sorbitol (previously obtained by milling of pure $\gamma$-sorbitol). Two fractions of $\alpha$-sorbitol were used: $X_{\alpha}=0.2$ and $X_{\alpha}=0.4$. Two interesting points can be noticed. First, the initial incubation time characteristic of the transformation $\gamma \rightarrow \alpha$ strongly shortens for increasing initial fractions of form $\alpha$. This clearly indicates that the conversion of $\gamma$-crystallites is strongly facilitated by the presence of preexisting $\alpha$-crystallites in their surroundings. Acceleration of transformations due to an addition of seeds of the final form is quite frequent. For instance, it often occurs for crystallization of liquids or polymorphic transformations which are recognize to be governed by a nucleation and grow process. In that case, adding seeds suppresses the 
nucleation stage and thus accelerates the transformation kinetics. On the contrary, transformations induced by milling cannot be thought to be governed by a nucleation and growth process. As a result, the decrease of the incubation time upon seeding is puzzling and must have a different origin. The preexisting crystallites (seeds) are expected to increase the probability to form a stable cluster of $\alpha$ crystallites and thus reduce the time required for its formation, i.e. the incubation time. This strongly suggests that the transformation of the pure form $\gamma$ (i.e. non-seeded - figure 5, black curve) is triggered by a small fraction of $\alpha$ crystallites produced during the incubation stage, even if this fraction is too weak to give a direct $\mathrm{X}$-ray diffraction signature. The second interesting point concerns the decrease of the initial fraction of form $\alpha$ during the first 10 minutes of milling. This decrease is attributed to the fact that most of $\alpha$-seeds are not yet involved in a stable cluster, so that they are expected to be likely converted into the form $\gamma$ after impact. So, both the decrease of the incubation time induced by seeding with form $\alpha$, and the disappearance of most of the seeds in the early milling stage can be understood in the frame of the proposed scenario. This scenario will be further detailed and strengthened by numerical simulations presented in the next section.

Moreover, the evolution of the average crystallite size of forms $\gamma$ and $\alpha$ during milling could be derived from the Rietveld analysis when their faction in the sample was greater than 0.2. These evolutions are reported in Figure 4. It appears that the crystallite size of the form $\gamma$ almost halves (100 nm to $60 \mathrm{~nm}$ ) in the very beginning of the milling. It then remains constant during the incubation time and during the conversion stage toward $\alpha$. Interestingly, the $\alpha$-crystallites generated by the milling appear to be much larger than the $\gamma$-crystallites (milled or not). In fact, $\alpha$-crystallites are so large that the broadening of Bragg peaks due to their finite size is too small to determine this size with accuracy. However, it can be said with certainty that the average crystallite size of $\alpha$-crystallites is higher than $200 \mathrm{~nm}$. The much larger size of $\alpha$ crystallites compare to $\gamma$ crystallites indicate that an $\alpha$-crystallite cannot arise from the mere conversion of a single $\gamma$-crystallite. The production of large $\alpha$-crystallites thus requires a redistribution of matter which can only be explained by a transient amorphization induced by mechanical impacts. Such an amorphous fraction can fuel the growth of $\alpha$-crystallites or allow the cold welding of two $\alpha$-crystallites through a coherent recrystallization between them. This latter mechanism was already reported ${ }^{36,54}$ for the milling of metallic compounds. Moreover, transient amorphization during polymorphic transformations induced by milling was already detected in a few pharmaceutical compounds ${ }^{26,55}$. The evolution of the crystallite size (figure 4) thus strongly suggests that mechanical shock induce a transient amorphization of the impacted fractions immediately followed by rapid recrystallizations.

The transformation $\gamma \rightarrow \alpha$ upon milling was also followed by Raman spectroscopy in an attempt to detect some signs of transformation toward the form $\alpha$ during the incubation time. The Raman spectrum of a crystalline phase in the molecular fingerprint region is dependent on the symmetry of the molecular conformation and also on the crystalline symmetry of the molecular materials. As a consequence, Raman spectroscopy is very sensitive to detect structural changes in the physical state of the sample and to provide a very fast characterization of the material just after milling. In particular, sample preparation and analyses can be done within a few seconds instead of at least 20 minutes for $X$-ray diffraction investigations. Such a fast investigation can thus limit any potential reverse transformation of the metastable form $\alpha$ toward the stable form $\gamma$ which could mask its formation during the incubation time. Figure 6 shows Raman spectra of the most intense Raman band of the molecular fingerprint region collected immediately after different milling times ranging from $\mathrm{tm}=0$ to $\mathrm{tm}=300 \mathrm{~min}$. At tm=0 $\mathrm{min}\left(\gamma\right.$-form), the band is located around $880 \mathrm{~cm}^{-1}$ with a shouldering on the left- 
wing. Upon milling, the main component of the band decreases while the left shouldering develops, so that after 300 min of milling the band appears to be centred around $870 \mathrm{~cm}^{-1}$ with a shouldering on the right-wing. These features are characteristic of the form $\alpha$ and thus confirm the transformation $\gamma \rightarrow \alpha$ induced by milling. Interestingly, a tiny decrease of the main component around $880 \mathrm{~cm}^{-1}$ and a slight development of the left-handed shouldering, can already be detected after 30 and 90 min of milling. Raman spectroscopy thus reveals the development of a tiny fraction of form $\alpha$ in the sample during the incubation time which could not be detected by X-ray diffraction and DSC. The very slight development of $\alpha$-crystallites during the incubation period is coherent with the shortening of the incubation time observed above when seeding the starting $\gamma$ form with $\alpha$-crystallites. It is also in line with what was observed by in-situ synchrotron X-ray diffraction during the milling induced transformation of mannitol ${ }^{32}$.

\subsection{MODELLING OF TRANSFORMATION KINETICS}

The simulation of the polymorphic transformation of sorbitol upon milling has been performed using the same model than that previously developed by Martinetto et al. ${ }^{32}$ in the case of mannitol. In this model, we consider that the initial powder sample is made of $\mathrm{N}$ grains which are themselves made of $\mathrm{n}^{3}$ crystallites in the form $\gamma$. The crystallites inside a grain are arrayed on a $\mathrm{n} \times \mathrm{n} \times \mathrm{n}$ cubic lattice. The position of crystallites inside the grains does not change during milling so that a crystallite is in contact with the same six neighbours during all the simulation. The milling process is simulated by impacting randomly the crystallites one after the other. Each impact is expected to induce an amorphization which is immediately (i.e. before the next impact) followed by a rapid recrystallization. The key point of the model is that the nature of this recrystallisation (toward $\gamma$ or $\alpha$ ) strongly depends on the structural state of the six neighbouring crystallites. More precisely, the nature of the transformation is driven by the number $\left(n_{\alpha}\right)$ of neighbouring crystallites in the structural state $\alpha$. If $n_{\alpha} \geq 3$ the recrystallization is expected to occur toward the new form $\alpha$ with a very high probability $\left(P_{\alpha}\right)$. On the contrary, if $n_{\alpha}<3, P_{\alpha}$ is very weak and the recrystallization is very likely to occur toward the starting form $\gamma$.

In practice, the simulation has been performed with $\mathrm{N}=100$ grains made of $\mathrm{n}^{3}=8000$ crystallites and using the probabilities $P_{\alpha}\left(n_{\alpha}<3\right)=0.05$ et $P_{\alpha}\left(n_{\alpha} \geq 3\right)=0.98$ which lead to a kinetic of transformation which best fit the experimental one. The transformation kinetics obtained with these parameters is reported in figure 7 using a reduced time scale which allows the comparison with the experimental kinetics. Clearly, this kinetics has a sigmoidal shape which mimics that of the experimental system. The specific sigmoidal shape arises directly from the recrystallization rule which is expected to induce a transformation in two stages. In the first stage, the number of $\alpha$-crystallites is very weak $\left(X_{\alpha}=0\right.$ at $t_{m}$ $=0$ ) so that the condition $n_{\alpha} \geq 3$ is never met. As a result, the balance between the $\alpha \rightarrow \gamma$ and $\gamma \rightarrow \alpha$ transitions will lead to a weak stationary fraction of form $\alpha$ close to $X_{\alpha}=P_{\alpha}\left(n_{\alpha}<3\right)$. This situation persists until a $2 \times 2 \times 2$ cubic cluster of $\alpha$-crystallites accidentally forms in the system giving rise to 8 crystallites characterized by $n_{\alpha} \geq 3$. These $\alpha$ crystallites have thus a high stability (i.e. a long life time) and their persistence will strongly facilitate the conversion of the neighbouring $\gamma$ crystallites toward the form $\alpha$ when impacted. This event thus marks the beginning of the second stage during which the $\gamma$-crystallites transform massively and rapidly towards the form $\alpha$. The succession of stages 1 and 2 thus gives rise to the sigmoidal kinetics of transformation observed experimentally. 
As for the experimental investigation, we have studied the effect of a seeding of the form $\gamma$ with crystallites of form $\alpha$ on the transformation $\gamma \rightarrow \alpha$ upon milling. The seeding was performed by replacing randomly a fraction $X_{\alpha}^{0}$ of the $8000 \gamma$-crystallites by $\alpha$-crystallites in the initial configuration. Two initial fractions of $\alpha$-crystallites have been considered: $X_{\alpha}^{0}=0.15$ and 0.20 . The kinetics of transformations of the three seeded samples are reported in figure 8 and compared to that of the nonseeded $\gamma$-form. It appears clearly that the effects of seeding are fully similar to those observed in the experimental system. We observe, in particular, an acceleration of the kinetics for increasing seeding levels which mainly appears through a shortening of the incubation time. This shortening is attributed to the higher number of $\alpha$-crystallites in the initial configuration, which decreases the time required to accidentally form a $2 \times 2 \times 2$ cubic cluster of $\alpha$-crystallites which is intrinsically more stable that an isolated $\alpha$-crystallite. We also observe the rapid decrease of $X_{\alpha}$ in the very early stage of the kinetics. This decrease is due to the rapid conversion of seeds ( $\alpha$-crystallites) which are not yet involved in a (at least) $2 \times 2 \times 2 \alpha$-cube, toward the form $\gamma$. It thus appears that the striking experimental features characterizing the kinetic of polymorphic transformation of sorbitol upon milling also clearly emerge from the simulation results. This strongly suggests that the model we have proposed captures most of the essential mechanisms which govern this kind of transformation. 


\section{CONCLUSION}

In this paper we have investigated the microscopic mechanisms which govern the polymorphic transformation $\gamma \rightarrow \alpha$ of sorbitol upon milling. This investigation was performed experimentally through a detailed analysis of the kinetics of this transformation, and by numerical simulations of a phenomenological model which captures the essentials features of both the experimental system and the milling perturbation.

The kinetics of transformation was determined by PXRD. It appears to be sigmoidal with a nearly 2hours incubation time which precedes a fast transformation stage occurring in less than 1 hour. We have shown that these specific kinetic features are likely to result from a two-step mechanism of transformation occurring after each ball impact. This mechanism consists of an amorphization of the impacted fraction, immediately followed by a fast recrystallization oriented towards the forms $\gamma$ or $\alpha$ by the surrounding crystallites. This leads to a two-stage transformation. In the first stage, the sample is mainly constituted of $\gamma$-crystallites so that recrystallizations mainly occur toward this very phase leading to a stationary metastable state characterized by a weak fraction of the final $\alpha$-form. The second stage starts when a stable cluster of $\alpha$-crystallites forms accidentally. At that point, recrystallizations will be increasingly oriented toward the final form $\alpha$. The combination of the two stages gives a sigmoidal shape to the overall kinetics. Such a scenario was confirmed experimentally by showing that seeding the starting form with the final one strongly decreases the incubation time of the transformation. It was also confirmed by numerical simulations of a phenomenological model able to reproduce the characteristic features of the experimental kinetics of transformation.

\section{ACKNOWLEDGEMENTS}

One of us (A.D.) thanks the region Hauts-de-France for the financial support which allowed him to conduct its $\mathrm{PhD}$ thesis and the present work. 


\section{CAPTIONS}

Table 1: $\quad$ Space groups, lattice parameters, cell volumes, Z numbers (number of molecules per cell) and refcodes of $\gamma$ and $\alpha$ forms used for Rietveld analyses ${ }^{45,46}$

Figure 1: Typical shapes of amorphization $9^{9,10,37,38}$ and polymorphic transformation ${ }^{32,39}$ kinetics induced by mechanical milling.

Figure 2: a) Evolution of the X-ray diffraction pattern of $\gamma$-sorbitol upon milling. The milling times are reported on the left-hand side of the figure. Dashed-lines mark characteristic peaks of $\gamma$-sorbitol and $\alpha$-sorbitol.

b) DSC curves of sorbitol recorded upon heating $\left(2^{\circ} \mathrm{C} / \mathrm{min}\right)$ after different milling times ranging from $t_{m}=0$ min to $t_{m}=300 \mathrm{~min}$. The milling times $t_{m}$ are reported on the left-hand side of the figure.

Figure 3: X-ray diffraction pattern of sorbitol milled for $140 \mathrm{~min}$ (black dots) compared to the bestfit profile obtained with Maud (red line). Fit parameters indicate that the fractions of form $\alpha$ and $\gamma$ are respectively $X_{\alpha}=0.60$ and $X_{\gamma}=0.40$.

Figure 4: Left axis: Time evolution of the fraction of form $\alpha\left(X_{\alpha}\right)$ during the transformation $\gamma \rightarrow \alpha$ upon milling, determined by PXRD (black circle). The solid black line is a guide for eyes.

Right axis: Time evolution of the average crystallite size of the form $\gamma$ (blue line), and the form $\alpha$ (red line), during milling. Crystallite sizes are derived from PXRD analysis. The solid lines are guides for eyes.

Figure 5: Experimental kinetics of transformation of sorbitol upon milling. The three curves correspond to initial samples of $\gamma$-form seeded by 0,20 and $40 \%$ of form $\alpha$. The transformed fractions are derived from PXRD analysis.

Figure 6: Raman spectra of the most intense Raman band of the molecular fingerprint region recorded immediately after different milling times ranging from $t_{m}=0$ min to $t_{m}=300 \mathrm{~min}$. The spectrum of the quenched liquid (grey line) is also reported for comparison.

Figure 7: Kinetics of transformation $(\gamma \rightarrow \alpha)$ of sorbitol upon milling. In black: experimental data determined by X-ray diffraction. In red: data obtained by numerical simulations (see text for details). The time scale has been normalized by the half completion time $\left(t_{1 / 2}\right)$ to compare experimental and simulation data.

Figure 8: Simulated kinetics of transformation of sorbitol upon milling. The three curves correspond to initial samples of $\gamma$-form seeded by 0,15 and $20 \%$ of form $\alpha$. See text for model and simulation details. A time step corresponds to 8000 impacts so that each crystallite is impacted on average once per unit of time. 
Table 1

\begin{tabular}{|c|c|c|c|c|c|c|c|}
\hline Sorbitol & Space group & $\mathrm{a}(\AA)$ & $\mathrm{b}(\AA)$ & $\mathrm{c}(\AA)$ & $\mathrm{V}\left(\AA^{3}\right)$ & $\mathrm{Z}$ & Ref code \\
\hline $\mathrm{\gamma}$ form & $\begin{array}{c}\text { orthorhombic } \\
\mathrm{P} 2_{1} 2_{1} 2\end{array}$ & 24.301 & 20.572 & 4.867 & 2433 & 12 & 254343 \\
\hline$\alpha$ form & $\begin{array}{c}\text { triclinic } \\
\mathrm{P} 2_{1} 2_{1} 2_{1}\end{array}$ & 8.677 & 9.311 & 9.727 & 785 & 4 & 1495366 \\
\hline
\end{tabular}


Figure 1

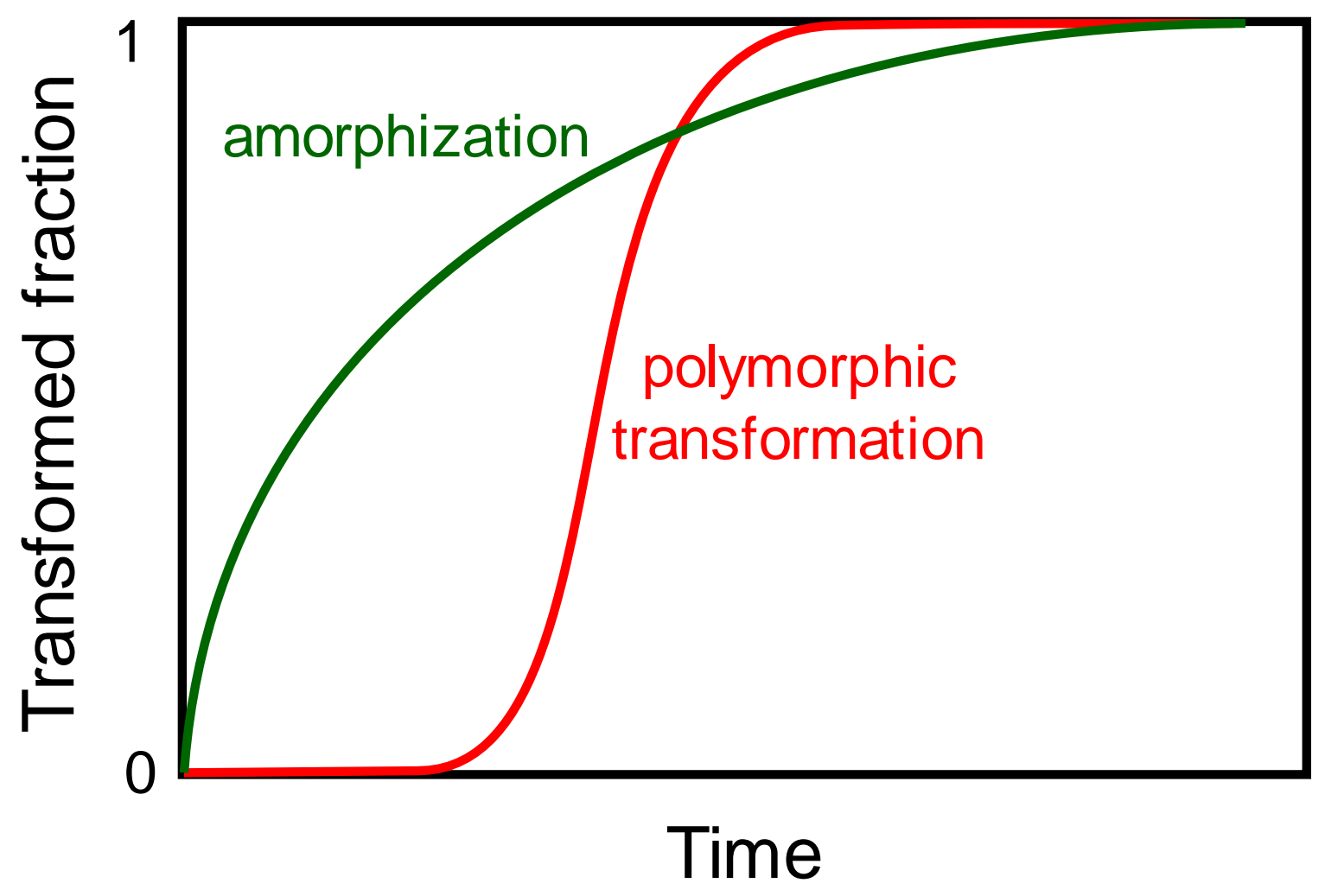


Figure 2
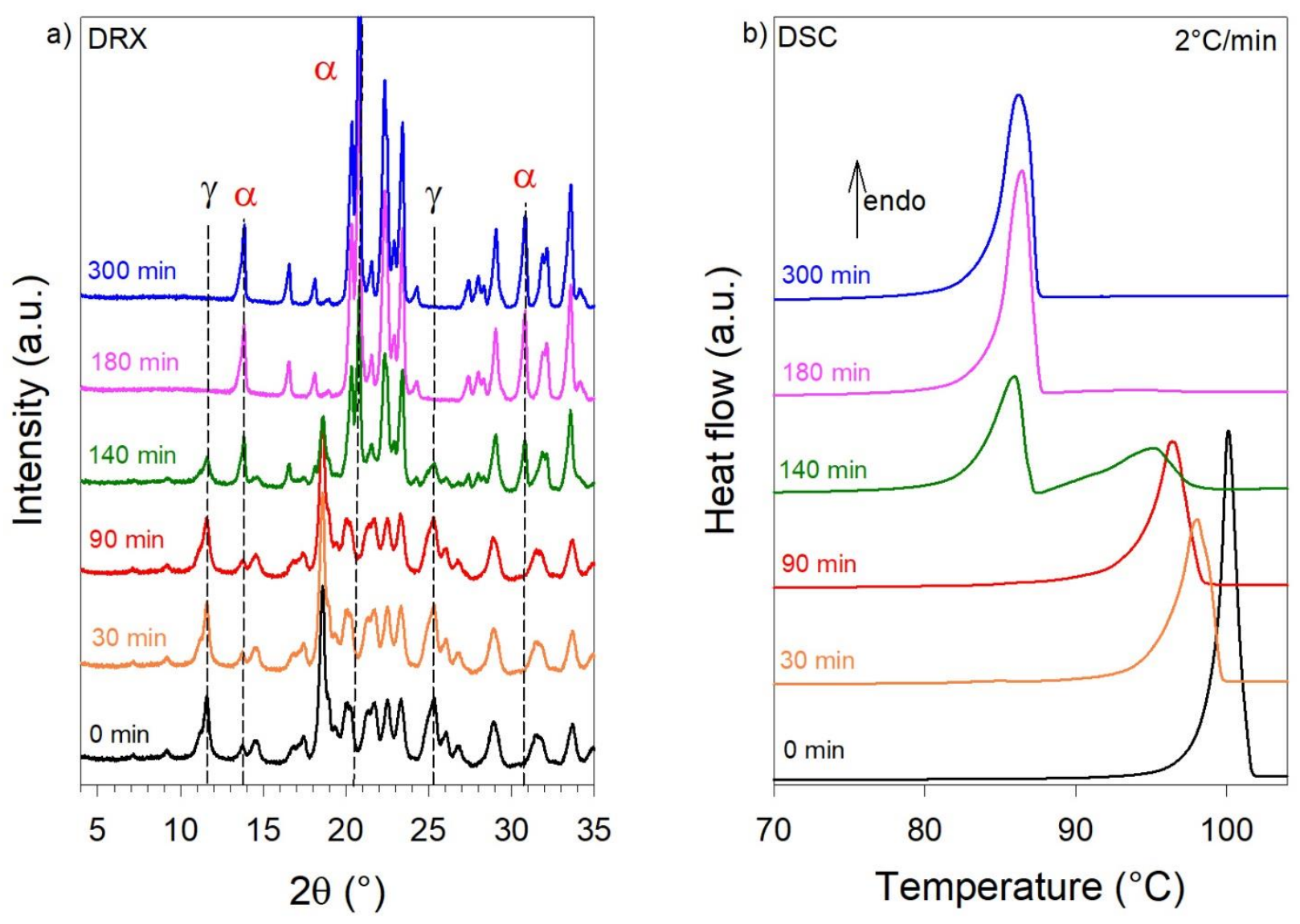
Figure 3

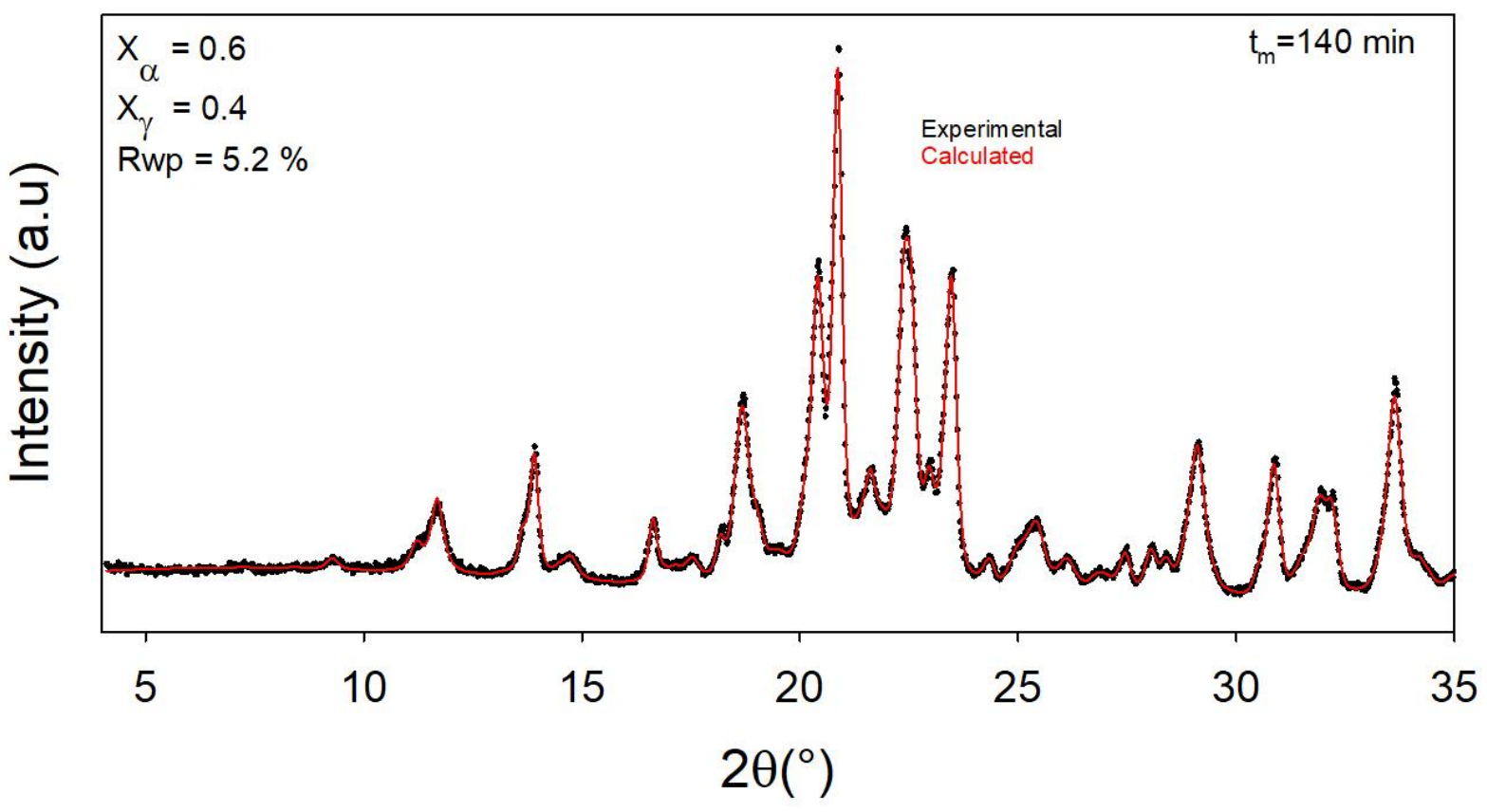


Figure 4

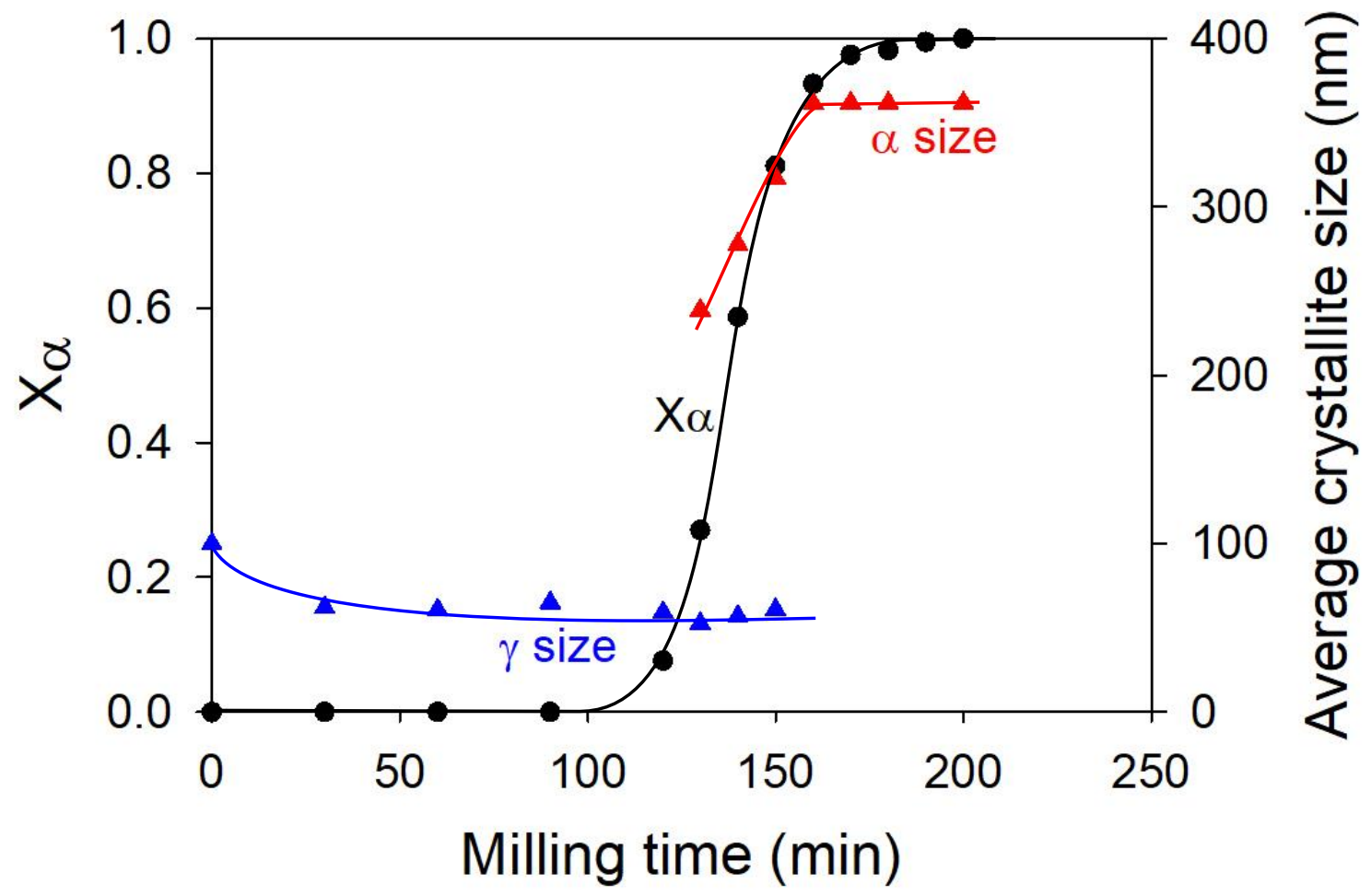


Figure 5

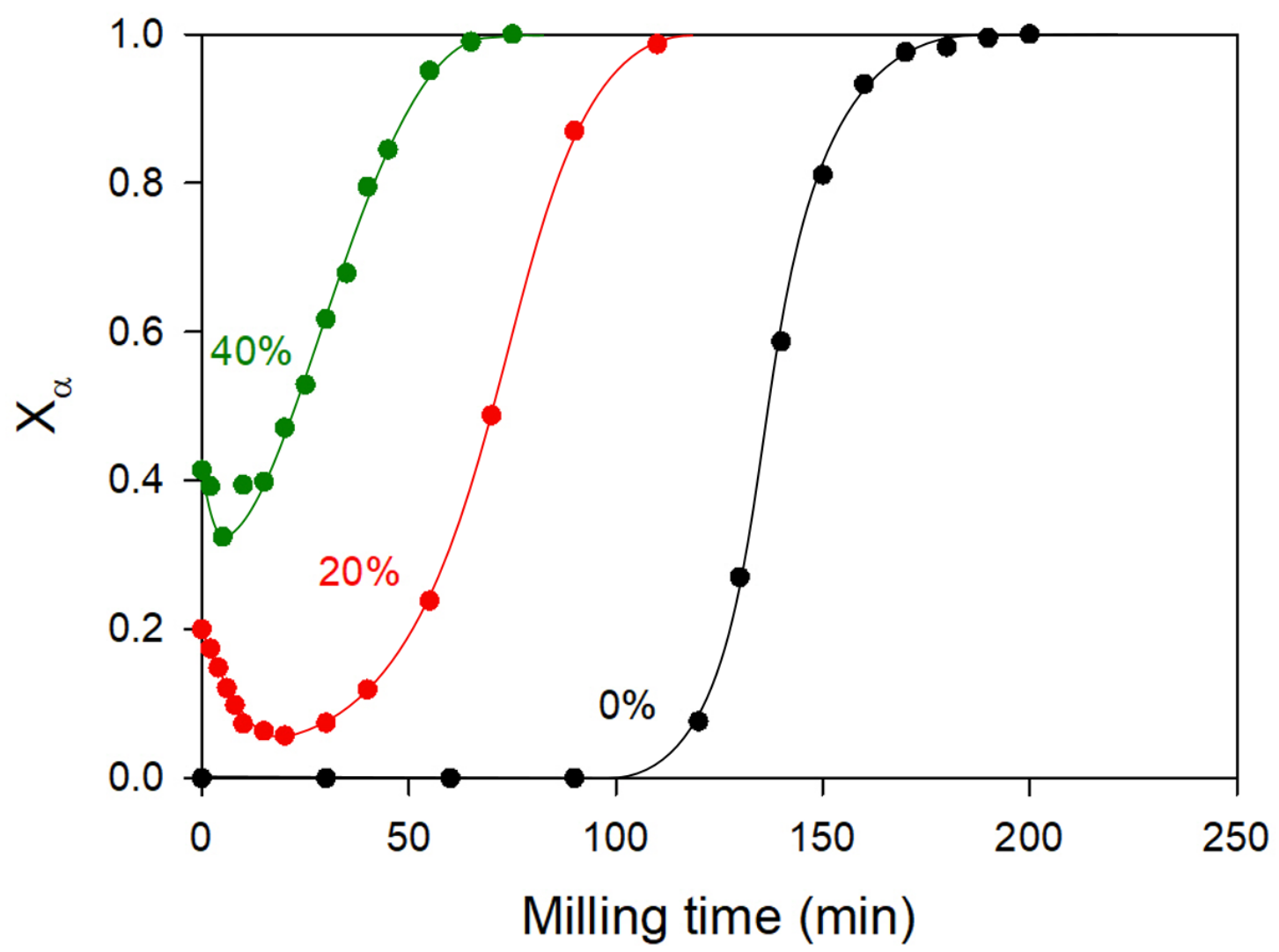


Figure 6

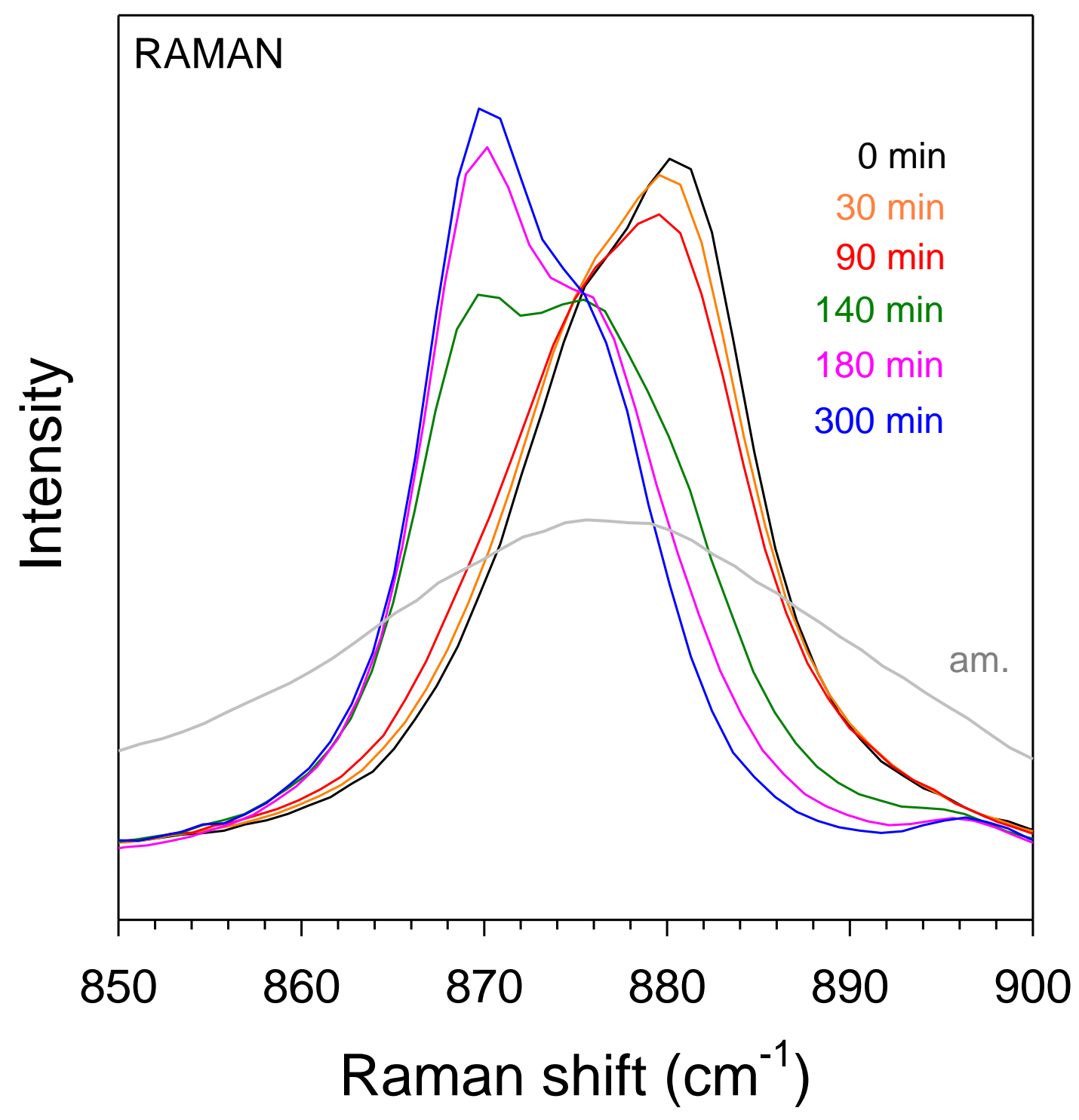


Figure 7

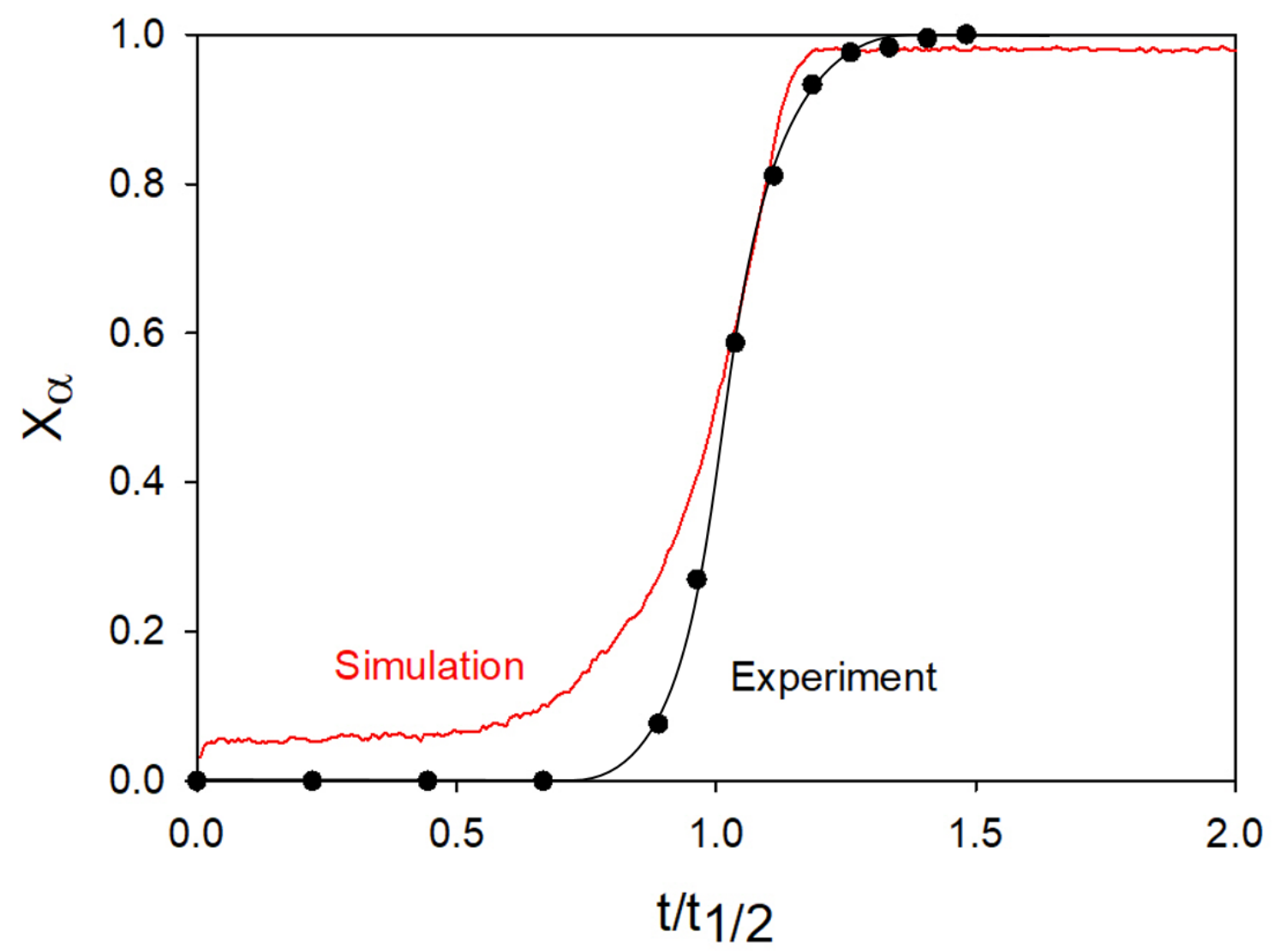


Figure 8

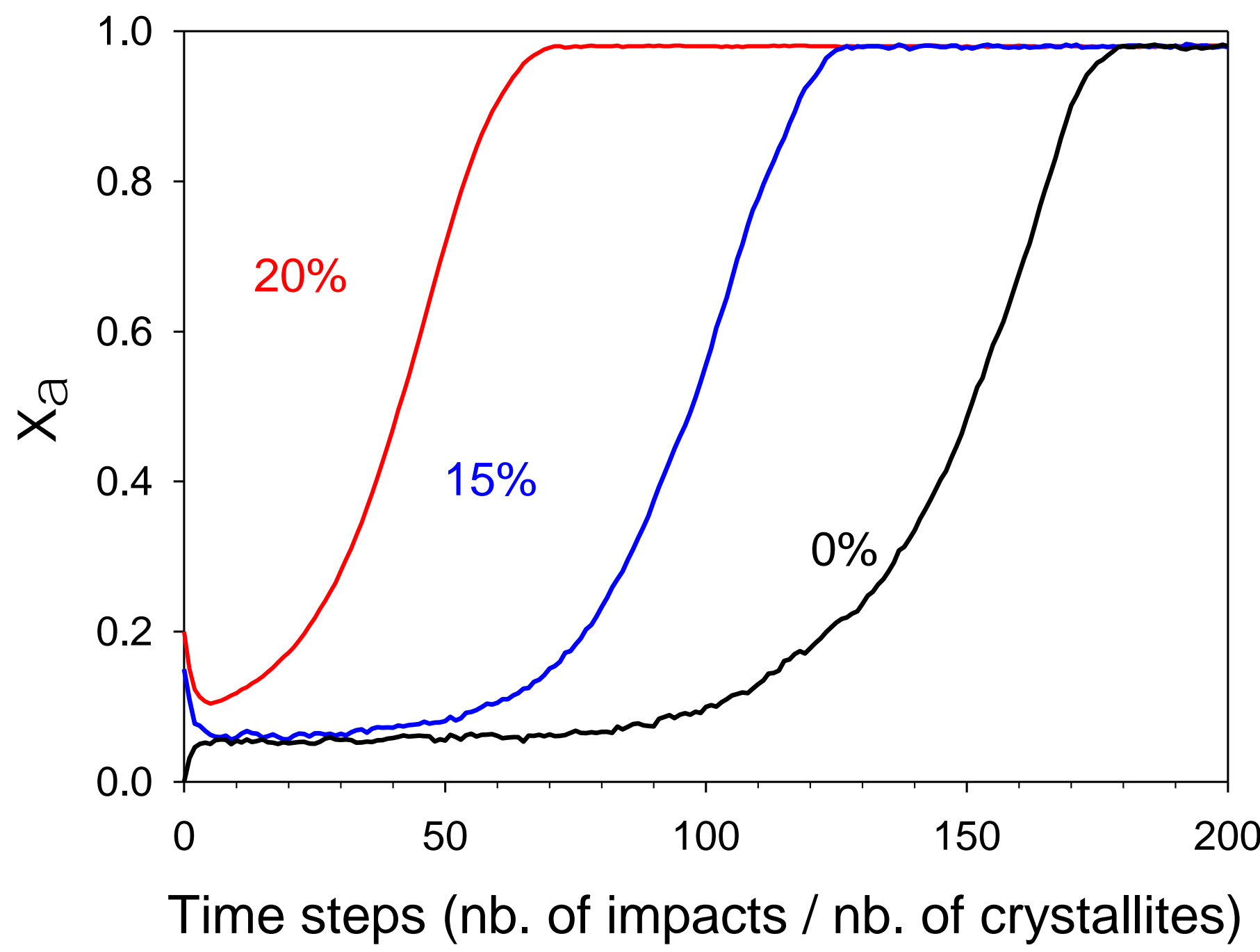




\section{REFERENCES}

1. Brittain HG. Effects of mechanical processing on phase composition. J Pharm Sci. 2002;91(7):1573-1580. doi:10.1002/jps.10115

2. Willart JF, Descamps M. Solid State Amorphization of Pharmaceuticals. Mol Pharm. 2008;5(6):905-920. doi:10.1021/mp800092t

3. Willart JF, Caron V, Lefort R, Danede F, Prevost D, Descamps M. Athermal character of the solid state amorphization of lactose induced by ball milling. Solid State Commun. 2004;132(10):693-696. doi:10.1016/j.ssc.2004.09.007

4. Dujardin N, Willart J, Dudognon E, et al. Solid state vitrification of crystalline $\alpha$ and $\beta$-Dglucose by mechanical milling. Solid State Commun. 2008;148(1-2):78-82. doi:10.1016/j.ssc.2008.07.002

5. Nagahama M, Suga $\mathrm{H}$. Molecular alloys formed by solid-state vitrification. J Mol Liq. 2002;95(3):261-284. doi:10.1016/s0167-7322(01)00292-6

6. Nagahama $\mathrm{M}$, Suga $\mathrm{H}$, Andersson $\mathrm{O}$. Formation of molecular alloys by solid-state vitrification. Thermochim Acta. 2000;363(1-2):165-174. doi:10.1016/s0040-6031(00)00607-9

7. Patterson JE, James MB, Forster AH, Lancaster RW, Butler JM, Rades T. Preparation of glass solutions of three poorly water soluble drugs by spray drying, melt extrusion and ball milling. Int J Pharm. 2007;336(1):22-34. doi:10.1016/j.ijpharm.2006.11.030

8. Ma E. Alloys created between immiscible elements. Prog Mater Sci. 2005;50(4):413-509. doi:10.1016/j.pmatsci.2004.07.001

9. Elisei E, Willart JF, Danède F, Siepmann J, Siepmann F, Descamps M. Crystalline Polymorphism Emerging From a Milling-Induced Amorphous Form: The Case of Chlorhexidine Dihydrochloride. J Pharm Sci. 2018;107(1):121-126. doi:10.1016/j.xphs.2017.07.003

10. Oliveira PFM, Willart J-F, Siepmann J, Siepmann F, Descamps M. Using Milling To Explore Physical States: The Amorphous and Polymorphic Forms of Dexamethasone. Cryst Growth Des. 2018;18(3):1748-1757. doi:10.1021/acs.cgd.7b01664

11. Latreche $M$, Willart JF, Guerain $M$, Hédoux A, Danède F. Using Milling to Explore Physical States: The Amorphous and Polymorphic Forms of Sulindac. J Pharm Sci. 2019;108(8):26352642. doi:10.1016/j.xphs.2019.03.017

12. Karki S, Frisi T, Jones W, Motherwell WDS. Screening for Pharmaceutical Cocrystal Hydrates via Neat and Liquid-Assisted Grinding . Mol Pharm. 2007;4(3):347-354.

13. Friščić $T$, Jones W. Recent Advances in Understanding the Mechanism of Cocrystal Formation via Grinding. Cryst Growth Des. 2009;9(3):1621-1637. doi:10.1021/cg800764n

14. Friscic T, Fabian L, Burley JC, Jones W, Motherwell WDS. Exploring cocrystal?cocrystal reactivity via liquid-assisted grinding: the assembling of racemic and dismantling of enantiomeric cocrystals. Chem Commun. 2006;(48):5009. doi:10.1039/b613073a

15. Guerain M, Derollez P, Roca-Paixão L, Dejoie C, Correia NT, Affouard F. Structure determination of a new cocrystal of carbamazepine and $\langle\operatorname{scp}>\mathrm{DL}</ \mathrm{scp}\rangle$-tartaric acid by synchrotron powder X-ray diffraction. Acta Crystallogr Sect C Struct Chem. 2020;76(3):225230. doi:10.1107/S2053229620000868 
16. Loh ZH, Samanta AK, Sia Heng PW. Overview of milling techniques for improving the solubility of poorly water-soluble drugs. Asian J Pharm Sci. 2015;10(4):255-274.

doi:http://dx.doi.org/10.1016/j.ajps.2014.12.006

17. Kipp JE. The role of solid nanoparticle technology in the parenteral delivery of poorly watersoluble drugs. Int J Pharm. 2004;284(1-2):109-122. doi:10.1016/j.ijpharm.2004.07.019

18. Merisko-Liversidge $\mathrm{E}$, Liversidge GG. Nanosizing for oral and parenteral drug delivery: A perspective on formulating poorly-water soluble compounds using wet media milling technology. Adv Drug Deliv Rev. 2011;63(6):427-440. doi:10.1016/j.addr.2010.12.007

19. Liversidge GG, Cundy KC. Particle size reduction for improvement of oral bioavailability of hydrophobic drugs. I: Absolute oral bioavailability of nanocrystalline danazol in beagle dogs. Int J Pharm. 1995;125(1):91-97.

20. Fisher ES. Milling of Active Pharmaceutical Ingredients. In: Swarbrick J, ed. Encyclopedia of Pharmaceutical Technology. Vol 4. New York, NY, USA; London, UK: Taylor \& Francis; 2006:2339-2351.

21. Descamps $M$, Willart JF. Perspectives on the amorphisation/milling relationship in pharmaceutical materials. Adv Drug Deliv Rev. 2016;100:51-66.

doi:10.1016/j.addr.2016.01.011

22. Otsuka M, Kaneniwa N. A kinetic study of the crystallization process of noncrystalline indomethacin under isothermal conditions. Chem Pharm Bull. 1988;36(10):4026-4032. http://www.scopus.com/inward/record.url?eid=2-s2.00023743976\& partnerID=40\&md5=70b97b85d2fd921b9e66ac7427255aaf.

23. Qi S, Weuts I, De Cort S, et al. An investigation into the crystallisation behaviour of an amorphous cryomilled pharmaceutical material above and below the glass transition temperature. J Pharm Sci. 2010;99(1):196-208. doi:10.1002/jps.21811

24. Linol J, Coquerel G. Influence of high energy milling on the kinetics of the polymorphic transition from the monoclinic form to the orthorhombic form of ( \pm )5-methyl-5-(4'methylphenyl)hydantoin. J Therm Anal Calorim. 2007;90(2):367-370.

http://www.scopus.com/scopus/inward/record.url?eid=2-s2.036849045666\& partnerlD=40\& $\mathrm{rel}=\mathrm{R} 8.2 .0$.

25. Otsuka M, Ohtani H, Otsuka K, Kaneniwa N. Effect of humidity on solid-state isomerization of various kinds of lactose during grinding. Journal-of-pharmacy-and-pharmacology. 1993;45(1):2-5.

26. De Gusseme A, Neves C, Willart JF, Rameau A, Descamps M. Ordering and disordering of molecular solids upon mechanical milling: The case of fananserine. J Pharm Sci. 2008;97(11):5000-5012. doi:10.1002/jps.21472

27. Descamps M, Willart JF, Dudognon E, Caron V. Transformation of pharmaceutical compounds upon milling and comilling: The role ofTg. J Pharm Sci. 2007;96(5):1398-1407. doi:10.1002/jps.20939

28. Willart JF, Durand M, Briggner LE, Marx A, Danède F, Descamps M. Solid-state amorphization of linaprazan by mechanical milling and evidence of polymorphism. J Pharm Sci. 2013;102(7):2214-2220. doi:10.1002/jps.23573

29. Schammé B, Couvrat N, Malpeli $\mathrm{P}$, et al. Transformation of an active pharmaceutical ingredient upon high-energy milling: A process-induced disorder in Biclotymol. Int J Pharm. 2016;499(1):67-73. doi:https://doi.org/10.1016/j.ijpharm.2015.12.032 
30. Chieng N, Rades T, Saville D. Formation and physical stability of the amorphous phase of ranitidine hydrochloride polymorphs prepared by cryo-milling. Eur J Pharm Biopharm. 2008;68(3):771-780. doi:10.1016/j.ejpb.2007.09.001

31. Trasi NS, Byrn SR. Mechanically Induced Amorphization of Drugs: A Study of the Thermal Behavior of Cryomilled Compounds. AAPS PharmSciTech. 2012;13(3):772-784. doi:10.1208/s12249-012-9801-8

32. Martinetto P, Bordet P, Descamps M, Dudognon E, Pagnoux W, Willart J-F. Structural Transformations of $d$-Mannitol Induced by in Situ Milling Using Real Time Powder Synchrotron Radiation Diffraction. Cryst Growth Des. 2017;17(11):6111-6122. doi:10.1021/acs.cgd.7b01283

33. Matsuoka M, Hirata J, Yoshizawa S. Kinetics of solid-state polymorphic transition of glycine in mechano-chemical processing. Chem Eng Res Des. 2010;88(9):1169-1173. doi:10.1016/j.cherd.2010.01.011

34. Brittain HG. Polymorphism and solvatomorphism 2010. J Pharm Sci. 2012;101(2):464-484. doi:10.1002/jps.22788

35. Delogu F, Cocco G. Relating Single-Impact Events to Macrokinetic Features in Mechanical Alloying Processes. J Mater Synth Process. 2000;8(5):271-277. doi:10.1023/a:1011382008963

36. Delogu F, Takacs L. Information on the mechanism of mechanochemical reaction from detailed studies of the reaction kinetics. J Mater Sci. 2018;53(19):13331-13342. doi:10.1007/s10853-018-2090-1

37. Bordet P, Bytchkov A, Descamps M, et al. Solid State Amorphization of $\beta$-Trehalose: A Structural Investigation Using Synchrotron Powder Diffraction and PDF Analysis. Cryst Growth Des. 2016;16(8):4547-4558. doi:10.1021/acs.cgd.6b00660

38. Ngono F, Willart J-F, Cuello G, Jimenez-Ruiz M, Affouard F. Lactulose: A Model System to Investigate Solid State Amorphization Induced by Milling. J Pharm Sci. 2018:(under press). doi:https://doi.org/10.1016/j.xphs.2018.09.013

39. Otsuka M, Kaneniwa N. Effect of seed crystals on solid-state transformation of polymorphs of chloramphenicol palmitate during grinding. J Pharm Sci. 1986;75(5):506-511.

40. Willart JF, Lefebvre J, Danède F, Comini S, Looten $P$, Descamps M. Polymorphic transformation of the $\Gamma$-form of D-sorbitol upon milling: Structural and nanostructural analyses. Solid State Commun. 2005;135(8):519-524. doi:10.1016/j.ssc.2005.04.048

41. Belenguer AM, Cruz-Cabeza AJ, Lampronti GI, Sanders JKM. On the prevalence of smooth polymorphs at the nanoscale: implications for pharmaceuticals. CrystEngComm. 2019;21(13):2203-2211. doi:10.1039/C8CE02098A

42. Yu L. Growth Rings in D-Sorbitol Spherulites: Connection to Concomitant Polymorphs and Growth Kinetics. 2003. doi:10.1021/cg034062n

43. Yu L, Mishra DS, Rigsbee DR. Determination of the Glass Properties of D-Mannitol Using Sorbitol as an Impurity. J Pharm Sci. 1998;87(6):774-777. doi:10.1021/js970224o

44. Paufler P. R. A. Young (ed.). The Rietveld Method. International Union of Crystallography. Oxford University Press 1993. 298 p. Price $\mathrm{f}$ 45.00. ISBN 0-19-855577-6. Cryst Res Technol. 1995;30(4):494-494. doi:10.1002/crat.2170300412

45. Rukiah M, Lefebvre J, Hernandez O, Van Beek W, Serpelloni M. Ab initio structure determination of the $\Gamma$ form of $D$-sorbitol (D-glucitol) by powder synchrotron X-ray diffraction. 
J Appl Crystallogr. 2004;37(5):766-772. doi:10.1107/S0021889804016206

46. Park YJ, Jeffrey GA, Hamilton WC. Determination of the crystal structure of the A form of Dglucitol by neutron and X-ray diffraction. Acta Crystallogr Sect B Struct Crystallogr Cryst Chem. 1971;27(12):2393-2401. doi:10.1107/s0567740871005934

47. Lutterotti L. Total pattern fitting for the combined size-strain-stress-texture determination in thin film diffraction. 2009. doi:10.1016/j.nimb.2009.09.053

48. Nezzal A, Aerts L, Verspaille M, Henderickx G, Redl A. Polymorphism of sorbitol. J Cryst Growth. 2009;311(15):3863-3870. doi:10.1016/j.jcrysgro.2009.06.003

49. Jackson CL, McKenna GB. The melting behavior of organic materials confined in porous solids. J Chem Phys. 1990;93(12):9002. doi:10.1063/1.459240

50. Fecht HJ. Defect-induced melting and solid-state amorphization. Nature. 1992;356(6365):133135. doi:10.1038/356133a0

51. Descamps M, Willart JF, Dudognon E, Lefort R, Desprez S, Caron V. Phase transformations induced by grinding: What is revealed by molecular materials. In: Materials Research Society Symposium Proceedings. Vol 979. Cambridge University Press; 2006:116-131. doi:10.1557/proc-979-0979-hh06-06

52. OTSUKA M, MATSUMOTO T, KANENIWA N. Effect of environmental temperature on polymorphic solid-state transformation of indomethacin during grinding. Chem Pharm Bull (Tokyo). 1986;34(4):1784-1793. doi:10.1248/cpb.34.1784

53. MacFhionnghaile $P$, Hu Y, Gniado K, Curran S, McArdle P, Erxleben A. Effects of ball-milling and cryomilling on sulfamerazine polymorphs: A quantitative study. J Pharm Sci. 2014;103(6):1766-1778. doi:10.1002/jps.23978

54. Desré PJ. A thermodynamic model for the nanocrystalline-to-glass transition in mechanically driven binary alloys. Philos Mag A Phys Condens Matter, Struct Defects Mech Prop. 1996;74(1):103-112. doi:10.1080/01418619608239692

55. Desprez S, Descamps M. Transformations of glassy indomethacin induced by ball-milling. J Non Cryst Solids. 2006;352(42-49 SPEC. ISS.):4480-4485. doi:10.1016/j.jnoncrysol.2006.02.130 